\title{
From the Editor
}

I continue to write this From the Editor during the worldwide coronavirus pandemic. I'm in Boston, Massachusetts, USA, where currently we are on a voluntary shelter-in-place. The way our city's and state's leadership has approached COVID-19 embodies care and thoughtfulness, and I am proud to be part of this inspiring community.

At the university where I teach, we moved our oncampus courses to remote teaching with the majority of students moving to their respective homes for education. I've had many years of distance education experience so the transition to remote teaching was seamless for me, but this was not the case for most faculty. I was happy to be appointed in the role of Remote Teaching Coordinator for my College so I could provide support to our community. What I observed was that faculty readily volunteered to help and support each other. For example, those with confidence in using remote communication platforms or assessments shared their knowledge and creative approaches with others. The focus was always on supporting each other and our students so we could continue to provide excellence in teaching and learning. In this time of uncertainty, faculty and administration reached out to each other and to our students in a caring and timely manner. What was happening can be summed in a famous Mr. Rogers quote: "When I was a boy, and I would see scary things in the news, my mother would say to me, 'Look for the helpers.' You will always find people who are helping." There are many people in this helping role around the globe. My sincere gratitude is extended to all of you.

This issue of WORK contains 24 articles. Topics are varied, such as shift working disorders among nurses of Tehran hospital and its relationship with job satisfaction; lung function and functional exercise capacity in underground semi-precious stone mineworkers; the prevalence of performance-related musculoskeletal disorders in fine arts faculty students and academics; predicting the relationship between safety climate and safety performance in cement industry; reducing lumbar spine flexion using real-time biofeedback during patient handling tasks; the role of time in the relation between perceived job insecurity and perceived job performance; the dressergonomics challenge: local costume as a new type of work dress? among other interesting topics. All of these articles are advancing knowledge in their respective topics and we're proud to publish them in WORK.

I hope you are continuing to enjoy our website at workjournal.org and to reading the blogs. You can find the link to our webinar series, Learn at WORK, on the website, too. Here is a list of our upcoming Learn at WORK webinars:

\section{Wednesday, May 13, 2020 from 1pm-2pmEST} Workplace Discrimination for Persons with Hearing Loss: Before and After the 2008 ADA Amendments Act

Presenter: Brian McMahon btmcmaho@vcu.edu Registration: https://attendee.gotowebinar.com/regis ter/465337912229392651

Wednesday, June 10, 2020 from 11am-12pmEST Gender and Stress-Buffering of Social Capital toward Depression among Precarious Workers in South Korea

Presenters: Dr. Park \& Dr. Oh psk2016@yonsei.ac.kr Registration: https://attendee.gotowebinar.com/regis ter/7955259498453971723

\section{Wednesday, July 8, 2020 from 1pm-2pmEST}

A Conceptual Framework to Promote Career Development for Vocational Rehabilitation Consumers with Traumatic Brain Injuries

Presenter: David Strauser strauser@illinois.edu Registration: https://attendee.gotowebinar.com/regis ter/61045286798182412

\section{Wednesday, August 5, 2020 from 1pm-2pmEST}

Does Objectively Measured Prolonged Standing for Desk Work Result in Lower Ratings of Perceived Low Back Pain than Sitting? A Systematic Review and Meta-Analysis 
Presenter: Diana Decarvalho Diana.decarvalho@ med.mun.ca

Registration: https://attendee.gotowebinar.com/regis ter/739866173672625164

\section{Wednesday, September 9, 2020 from 1pm- 2pmEST}

Millennial Preferences in Training Messages: The Role of Teamwork and Corporate Social Responsibility to WORK

Presenters: Diana Schwerha schwerha@ohio.edu \& Tyler Clark tc142810@ohio.edu

Registration: https://attendee.gotowebinar.com/regis ter/2725235628413300749

\section{Wednesday, October 14, 2020 from 1pm-2pmEST}

Development of an online digital resource accessible for students with visual impairment or blindness: challenges and strategies

Presenter: Dr. Almeida, marga@ua.pt

Registration: https://attendee.gotowebinar.com/regis ter/8581833792537508621
If you missed any of the Learn at WORK webinars, you can find the recordings on our website at workjournal.org and at the Learn at WORK YouTube channel: https://www.youtube.com/channel/UCO JalCXvSg9fPHaFFs48PuQ

As always, I welcome hearing from you. Be safe and healthy.

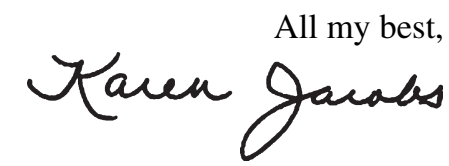

Founding Editor, WORK Occupational therapist \& ergonomist Kjacobs@bu.edu workjournal.org blogs.bu.edu/kjacobs/ @WORK_Journal https://www.facebook.com/ WORKJournal2016/?fref=ts 